\title{
The Development of Date Mining System Based on Data mining Algorithms of B/S Framework
}

\author{
Hongxia Liu \\ Department of Electronic Information Engineering, Handan Polytechnic College, Handan, 056001, \\ China
}

Keywords: Data algorithms; Data mining; Data storage; Agriculture data

\begin{abstract}
In this paper, it proposes a data mining system based on data mining algorithms of B/S framework, including the key parts such as visualization of data mining, processing of data mining processes and data storage etc... and initially achieves an agriculture data mining platform based on $\mathrm{B} / \mathrm{S}$ framework based on the chain of responsibility pattern. Finally, it combines the specific application cases of agriculture data to validate the expansibility, flexibility and effectiveness of the agricultural data mining platform based on Web.
\end{abstract}

\section{Introduction}

Data Mining is a process which extracts the information and knowledge that are implicit, novel and people do not know in advance, but are potentially useful from the data that is in a large number, incomplete, noisy, fuzzy and random. Data mining technology is completely application-oriented. It can not only be used for the simple search, query and call of a particular database, and can also do the statistics, analysis, synthesis and reasoning work for the data so as to guide solving the practical problems, find mutual relationships among the events and the forecast it. Therefore, the introduction of the data mining technology into the agriculture field data processing can effectively identify potential agricultural knowledge and useful data from the agriculture data, which provides planning, early warning, decision-making and other services for the agricultural sector, improves the agricultural environment and the quality of agricultural products and promotes agricultural production develop efficiently, coordinately and constantly[1-3]. The Ministry of Agricultural Environmental Monitoring Station based on the data warehouse of the agricultural environmental information decisive system including the agricultural environmental information and data warehouse, online analytical processing, data mining and other functional models [4-5].

Paper [1] adopts fuzzy evaluation algorithm toe evaluate the environment under the actual region conditions based on the comprehensive considering of each pollutant factor of the agricultural environment, and uses the APriori algorithm to find out the correlations among pollutant factors so as to dig out the specific reasons which causes the environmental differences. In summary, we learned that data mining technology embodies great advantages in the application of agricultural field, but, overall, the data mining technology in the field of agriculture is only at the exploratory stage[6-8]. In this paper, it provides the theoretical guidance for building agricultural data mining platform based on $\mathrm{B} / \mathrm{S}$ framework through presenting a "left-middle-right" principle framework of the three-tier framework for agricultural data mining platform and further analyzing its key technologies. It initially achieved an agricultural data mining platform based on $\mathrm{B} / \mathrm{S}$ framework by adopting the chain of responsibility pattern and applied it to the specific agricultural field data processing so as to achieve effective analysis of agricultural data, improve the quality and efficiency of agriculture and science activities, promote the intellectualization and practical application of the agricultural data mining, which established the foundation of the knowledge and application as well as the system decision-making of agriculture and promote the sustainable development of agricultural production. 


\section{The principle framework of data mining algorithms platform based on $\mathrm{B} / \mathrm{S}$ data algorithms}

Principle framework: The versatility, flexibility, expansibility and maintainability of data mining platform are the important factor of restricting application problems solving ability. The current existing data mining platforms for the field of application use relatively fixed processing procedures. It is much more difficult to deploy it if it is necessary to adjust and modify application modules for different applied data and data mining tasks. In response to these needs, it designs the following principle framework based on the Web agricultural data mining platform specifically including the "left-middle-right" three-tier which are the presentation layer, business logic layer and data layers.

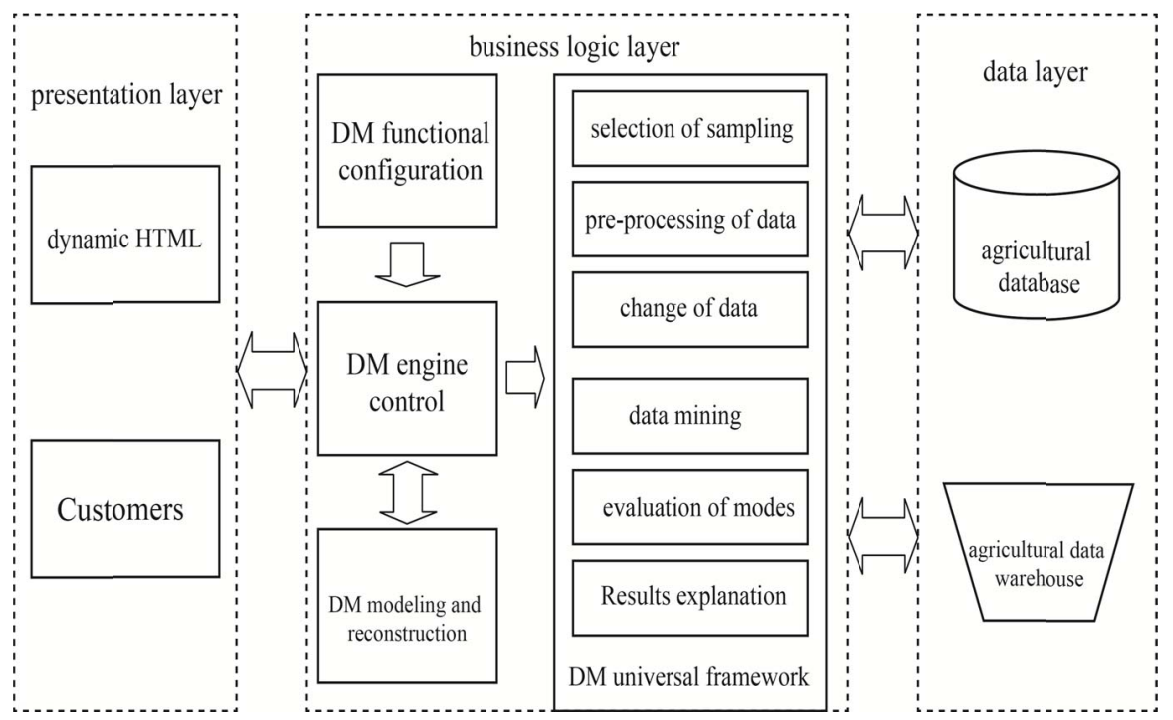

Figure 1 the principle framework of data mining platform based on $\mathrm{B} / \mathrm{S}$ data algorithms

Operating mechanism: Following the Multi-stage processes of model of the data mining and combining the agricultural data characteristics, it designs the original framework based on the $\mathrm{B} / \mathrm{S}$ of agricultural data mining platform. In Figure 2, it shows the "top-down" operating mechanism of the platform:

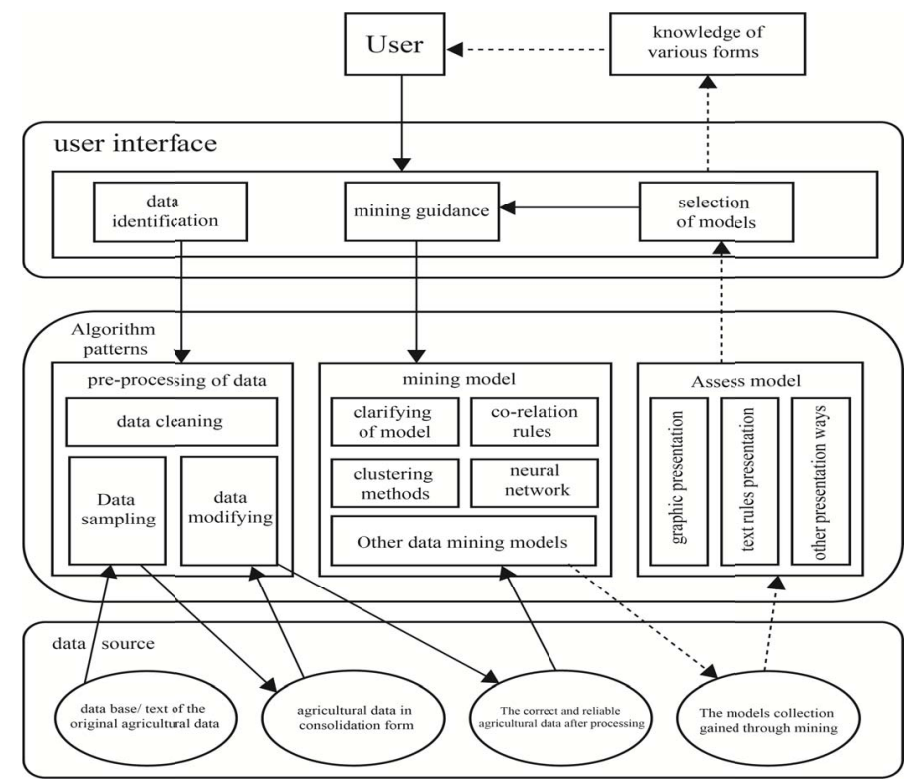

Figure 2 Operation mechanism of agricultural data mining platform based on Web

Data storage based on B/S: It is a critical to design reasonable data models for providing mining activities with the underlying data and build data analysis space if you want to fully and effectively utilize the massive number of agricultural data and ensure the efficiency of data mining due to the huge amount of data and various storage formats of agriculture. Since the dimension 
table of data is generally large and complex and it may have millions of pieces of data and varieties of attributes, in order to ensure the access performance of data, it joints the Specific Category Table which describes the fact table in details on dimension table, thereby reducing the fact table to minimize the amount of data storage and joint relatively smaller dimension tables to improve the query performance. In figure 3, it shows a schematic diagram of snowflake model of agricultural soil pollution monitoring data. The fact tables include testing results table, the monitoring unit table, points table; the dimension tables include historical monitoring data dimension tables, on-site survey dimension tables, crop samples dimension tables, data dimension tables in preliminary testing, testing data dimension tables of the intermediate state, time dimension tables, geographic data dimension tables and so on.

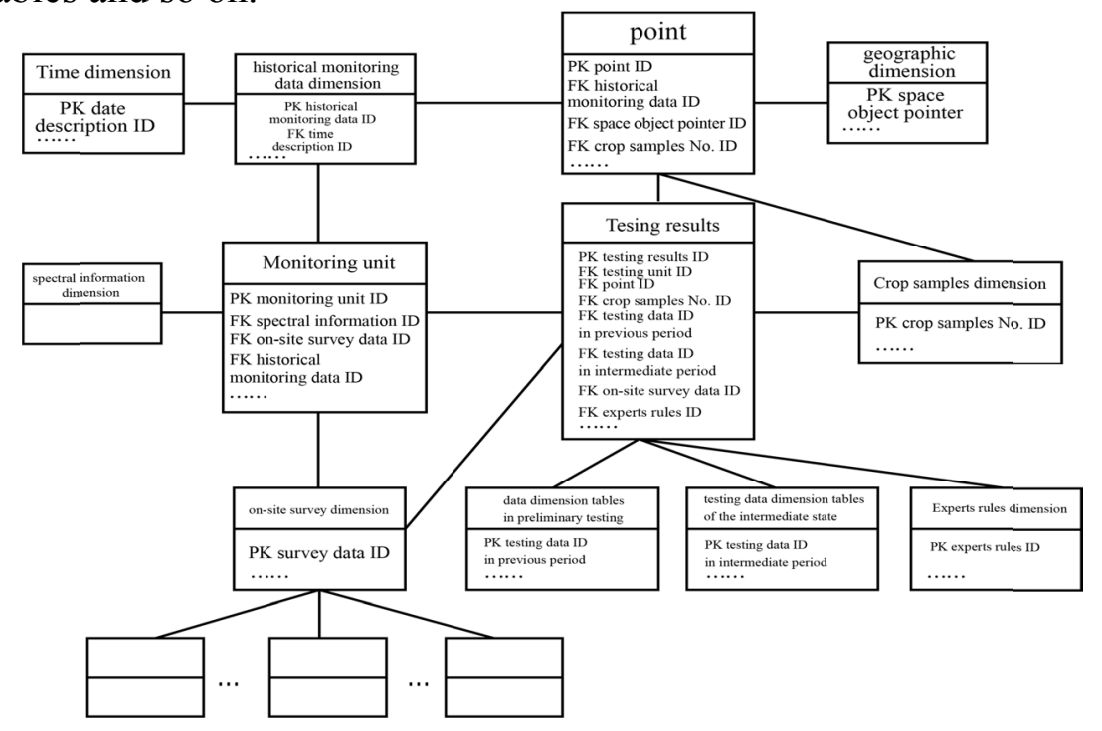

Figure 3 Snowflake schematic model of soil pollution monitoring data

\section{The initial achievement of agricultural data mining platform based on the B/S data algorithms}

The agricultural data mining platform based on the $\mathrm{B} / \mathrm{S}$ data algorithms has strong flexibility and expansibility and it is dynamically assembled according to the data mining processes needs by requiring for the function modules such as data preparation, data mining algorithms, evaluation results and so on. Therefore, it is very reasonable to choose the chain of responsibility structure to achieve the agricultural data mining algorithms platform based on the $\mathrm{B} / \mathrm{S}$.

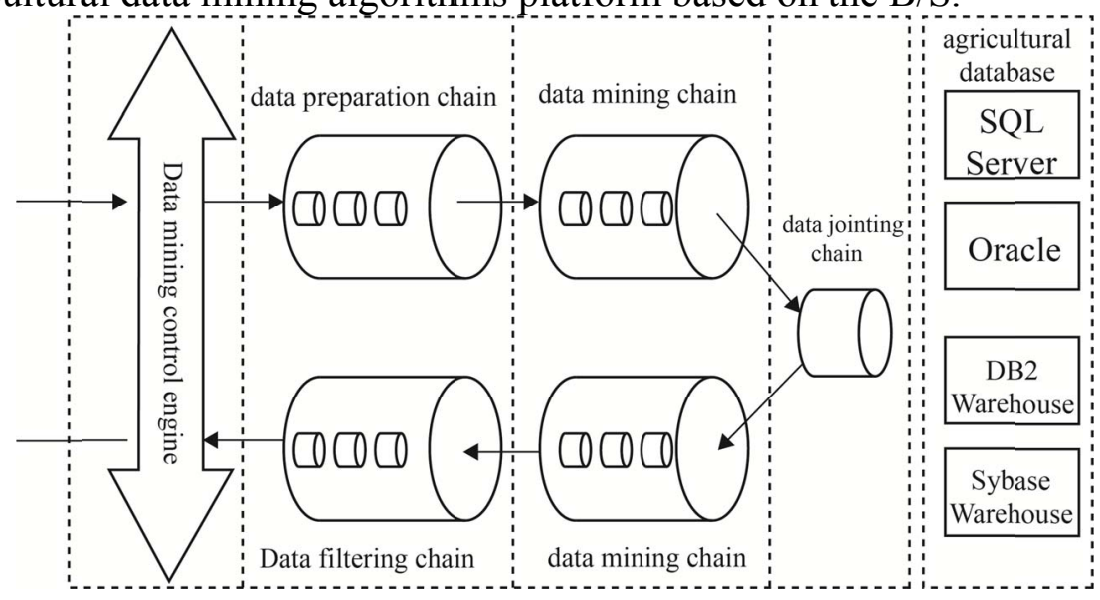

Figure 2 Chain of Responsibility models

The accomplishment of a data mining task involves at least the Data sample Handler, Data Transform Handler, data mining processor (such as Association Rule Handier), Data Provider Handler, Data Result Filter Handler, Data Visualization Handler) and so on. Under the driving of 
Data Mining Control Engine, they are charged for implementing respectively by Dataset of le Handler class, Data Transform Handler class, Association Rule Handler class, Data Provide Handler class, Data Result Filter Handier classes and Data Visualization Handler class. The interactive sequences of each Handler comply with the multi-stage processes model based on data mining and agriculture data mining principle framework, shown as in Figure 3.

\section{The data mining system of agricultural pests' data processing based on $\mathrm{B} / \mathrm{S}$ framework}

\section{The application of agricultural pests:}

Crop pests directly endanger the productivity and quality of agricultural products. China's annual economic losses caused by pests and diseases have been reached to $15 \%$ to $25 \%$ [50]. Take stem borer as an example, the stem borer is one of the most important pest in the production of rice. They seriously affect the rice productivity and quality. The amount of pests and diseases occurred is mainly affected by temperature, humidity and rainfall. Especially with the changes of weather conditions in winter, which overwintering, the amount of pests occurred changes quite differently. Therefore, based on the data of agricultural pests and diseases, mining the potential rules between the agricultural pests and climate has great significance on the preventing and controlling of the occurrence of pests.

Table 1 Discrete data

\begin{tabular}{|c|c|c|c|c|c|}
\hline No & $\begin{array}{l}\text { T. Avg. Jan } \\
\text { Nominal }\end{array}$ & R. Jan. Nominal & $\begin{array}{l}\text { T. Avg. Apr } \\
\text { Nominal }\end{array}$ & R. Apr. Nominal & Chile suppressal Nominal \\
\hline 1 & $(1-1.5]^{\prime}$ & $(30.27-\ldots$ & $(15.95-\ldots$ & $(71.1-1 \ldots$ & ${ }^{\prime}(-\inf -131]^{\prime}$ \\
\hline 2 & $(-$ inf--... & $(30.27-\ldots$ & $(17.35-\ldots$ & $(130.1 \ldots$ & '(131-224] \\
\hline 3 & $(-$ inf--... & $(68.63-\ldots$ & $(-$ inf-1... & $(159.6 \ldots$ & ${ }^{\prime}(224-317]^{\prime}$ \\
\hline 4 & $(1-1.5]^{\prime}$ & $(20.68-\ldots$ & $(14.9-1 \ldots$ & $(218.6 \ldots$ & '(224-317]' \\
\hline 5 & $(-\inf --\ldots$ & $(87.81-\ldots$ & $(-\inf -1 \ldots$ & $(277.6 \ldots$ & ${ }^{\prime}(224-317]^{\prime}$ \\
\hline 6 & $(1-1.5]^{\prime}$ & $(30.27-\ldots$ & $(15.6-1 \ldots$ & $(71.1-1 \ldots$ & $(-\inf -131]^{\prime}$ \\
\hline 7 & (3.5-inf) & $(20.68-\ldots$ & $(15.6-1 \ldots$ & $(71.1-1 \ldots$ & $(-\inf -131]^{\prime}$ \\
\hline 8 & $(2.5-3]^{\prime}$ & $(20.68-\ldots$ & $(17.35-\ldots$ & (-inf-4... & '(134-224]' \\
\hline
\end{tabular}

In figure 5, it shows a presentation of visualization of discrete data. Each two-dimensional data is corresponding to a column chart, a data dimension value is the specific intervals (grades); another data dimension is the "appearance" which indicates the number of records. Take ChifoS, namely pressalis for example, horizontal dimension is corresponding to $<131,131---224,224---317$, $>317$ four grades; vertical dimension is corresponding to Chilo SuPPressalis which takes a specific value in specific intervals of number of records, they are respectively 5,4,5,1.

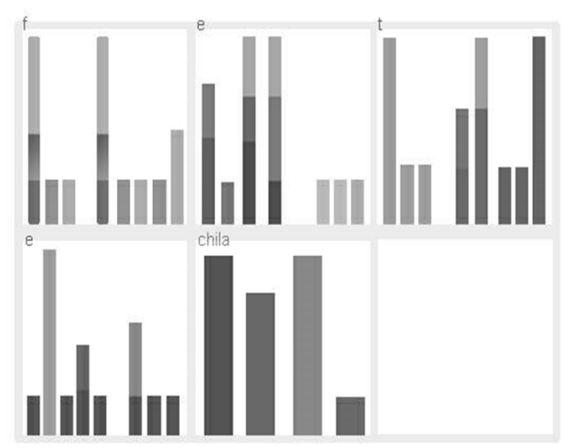

Figure 5 Visualization of discrete data

As for the modification of data mining processes in the data mining system of agricultural pests data processing based on $\mathrm{B} / \mathrm{S}$ framework, it can be modified by using the appointed APriori algorithms in the Association Rule Handier. After using the APriori mining algorithms to do the relevant rules of discovery, we can obtain the average temperature in January, the rainfall in January, the average temperature in April, the rainfall in and the potential rules of overwintering 
stem borer. The specific mining results can be shown as in Table 2:

Table 2 the predictive results of GA-BP algorithm based on Map Reduce

\begin{tabular}{|c|c|}
\hline 1 & Chilo suppressalis $=^{\prime}(- \text { inf-131] }]^{\prime} 5==>$ R. Apr $=^{\prime}(71.1-100.6]^{\prime} 5 \quad \operatorname{conf}(1)$ \\
\hline 2 & R. Apr. $={ }^{\prime}(71.1-100.6]^{\prime} 5==>$ Chilo suppressalis $=^{\prime}(- \text { inf-131] }]^{\prime} 5 \quad \operatorname{conf}(1)$ \\
\hline 3 & R. Apr. $={ }^{\prime}(218.6-248.1]^{\prime} 3==>$ T. Avg. Jan. $={ }^{\prime}(1-1.5]^{\prime} 3 \operatorname{conf}(1)$ \\
\hline 4 & T. Avg. Apr. $={ }^{\prime}(- \text { inf-14.55] }]^{\prime} 3==>$ Chilo suppressalis $={ }^{\prime}(224-317]^{\prime} 3$ conf(1) \\
\hline 5 & T. Avg. Apr. $={ }^{\prime}(17.35 \text {-inf })^{\prime} 3==>$ Chilo suppressalis $={ }^{\prime}(131-224]^{\prime} 3 \quad \operatorname{conf}(1)$ \\
\hline 6 & T. Avg. Jan. $={ }^{\prime}(3.5-\text { inf })^{\prime} 2==>$ R. Apr. $={ }^{\prime}(71.1-100.6]^{\prime} 2 \quad \operatorname{conf}(1)$ \\
\hline 7 & T. Avg. Jan. $={ }^{\prime}(3.5-\mathrm{inf})^{\prime} 2==>$ Chilo suppressalis $=^{\prime}(- \text { inf-131] }]^{\prime} 2 \operatorname{conf}(1)$ \\
\hline 8 & T. Avg. Jan. $={ }^{\prime}(- \text { inf-0.5] }]^{\prime}$ Chilo suppressalis $=^{\prime}(224-317]^{\prime} 2==>$ T. Avg. Apr. $={ }^{\prime}(-i n f-14.55]^{\prime} 2$ conf(1) \\
\hline 9 & T. Avg. Jan. $={ }^{\prime}\left(-\right.$ inf-0.5] ${ }^{\prime}$ T. Avg. Apr. $={ }^{\prime}\left(-\right.$ inf-14.55]' 2 Chilo suppressalis $={ }^{\prime}(224-317]^{\prime} 2$ conf $(1)$ \\
\hline 10 & R.Jan. $={ }^{\prime}(30.27-39.86]^{\prime}$ R. Apr. $={ }^{\prime}(218.6-248.1]^{\prime} 2==>$ T. Avg. Jan $=^{\prime}(1-1.5]^{\prime} 2 \operatorname{conf}(1)$ \\
\hline
\end{tabular}

\section{Conclusion}

The main innovative features of this paper are: It guarantees the expansibility of the system architecture through the framework of principle of agriculture data mining platform based on Web. It ensures the flexibility of data mining through the achievement of chain of responsibility and can dynamically adjust the data mining processes and replace the data mining algorithm.

\section{References}

[1] Liang Chuan. Research on the Data Mining System Building in Agricultural Information Grid [D]. Beijing: The academy thesis of China Agricultural Sciences of Masters, 2009.

[2] Jiawei Han, Micheline Kamber.Data Mining Concepts and Techniques.Translated by Fan Ming and Meng Xiaofeng. Data Mining ---Concepts and Techniques [M]. Beijing: China Machine Press, 2001:33-34

[3] CART. Salford System CART0. http://salford-systems.com/cart.php

[4] $\mathrm{Pu}$ Xiaoping. The Typical Application Based on Components Automatic System [J]. Programmable Controller \& Factory Automation, 2007 (8): 61-63

[5] Jiawei Han, Yonian Fu, Wei Wang, Jenny Chiang, Wan Gong, Krzysztof Koperski, Deyi Li, un Lu, Amynmohamed Raj an, Neboj sa Stefanovic Xia, Osmar R. Zaiane. DBMiner: A System for Relational Databases Proc. 1996 Int'1 Conf. On Mining Knowledge in Betty Large Data Mining and Knowledge Discovery (KDD'96) , Portland, Oregon, August 1996, 250-255.

[6]Zhu Tingxun, Gao Wen. KDD: Discovering of Knowledge in Database http://www.doc88.com/p-99950851955.htm1

[7] Jiawei Han ,Yonnian Fu ,Yue Huang, Yandong Cai and Nick Cercone .School of Computing Science, Simon Fraser University Canada. DBLearn: A System Prototype for Knowledge Discovery in Relational Databases.

[8] Yang Jie, Ye Chenzhou, Chen Nianyi. DB Mine: The Platform and Application of Data Mining [J]. Journal of System Simulation, 2001, 13 (6):740-742 